\title{
Simulating Space Capsule Water Landing with Explicit Finite Element Method
}

\author{
John T. Wang ${ }^{1}$ and Karen H. Lyle ${ }^{2}$ \\ NASA Langley Research Center, Hampton, VA, 23681
}

\begin{abstract}
A study of using an explicit nonlinear dynamic finite element code for simulating the water landing of a space capsule was performed. The finite element model contains Lagrangian shell elements for the space capsule and Eulerian solid elements for the water and air. An Arbitrary Lagrangian Eulerian (ALE) solver and a penalty coupling method were used for predicting the fluid and structure interaction forces. The space capsule was first assumed to be rigid, so the numerical results could be correlated with closed form solutions. The water and air meshes were continuously refined until the solution was converged. The converged maximum deceleration predicted is bounded by the classical von Karman and Wagner solutions and is considered to be an adequate solution. The refined water and air meshes were then used in the models for simulating the water landing of a capsule model that has a flexible bottom. For small pitch angle cases, the maximum deceleration from the flexible capsule model was found to be significantly greater than the maximum deceleration obtained from the corresponding rigid model. For large pitch angle cases, the difference between the maximum deceleration of the flexible model and that of its corresponding rigid model is smaller. Test data of Apollo space capsules with a flexible heat shield qualitatively support the findings presented in this paper.
\end{abstract}

\section{Introduction}

Many practical engineering problems require detailed water landing impact analyses. Examples of such problems include ship slamming, torpedo water entry, and space capsule sea landing. Analytical approaches for a rigid body water landing developed by von Karman ${ }^{1}$ and Wagner ${ }^{2}$ in the early nineteenth century are still being widely used for obtaining closed form solutions. A review describing water entry studies related to aerospace structures between 1929 and 2003 was recently published ${ }^{3}$. This review pointed out that finite element techniques for analyzing both fluid and structural response in a single model were only recently available. It also stated that the aircraft industry requires a validated numerical tool in which the interaction between the fluid and the structure can be modeled accurately. One final suggestion of the paper is that numerical modeling techniques such as hybrid analysis, finite element modeling and smoothed particle hydrodynamics ${ }^{4,5}$ will certainly be utilized for future work; however a large amount of work needs be done to fully validate these numerical modeling techniques.

Recent advancement in the high-speed computing technology for large-scale analyses and the development of the advanced explicit nonlinear dynamic finite element method provides a powerful capability for solving the fluid and flexible structure interaction (hydroelastic) problems. Explicit nonlinear dynamic finite element codes with an Arbitrary Lagrangian Eulerian (ALE) solver $^{6,7}$ for modeling water landing impact are currently available in commercial codes such as LS-DYNA ${ }^{\circledR 8}$ and MSC.Dytran ${ }^{\circledR 9}$. These codes have been used by both researchers and engineers for simulating water impact of aircraft, spacecraft, and ship hull structures ${ }^{10-12}$. These studies found that the computational methods implemented in the explicit nonlinear dynamic finite element codes can produce useful results and provide valuable insight into water impact problems. However, these studies also revealed the shortcomings of the codes such as the mesh dependency of the prediction results, the lack of good correlations between test data and numerical predictions, and the computationally intensive issues related to the explicit method and the ALE solver.

\footnotetext{
${ }^{1}$ Aerospace Engineer, Durability, Damage Tolerance \& Reliability Branch, MS188E, AIAA Associate Fellow.

2 Aerospace Engineer, Structural Dynamics Branch, MS495.
} 
In the Apollo program, a vast amount of test data and analytical solutions related to water landing impact was developed and documented in many publications, such as Refs. 13 to 16. Water landing was used exclusively for returning the Apollo space capsule to the earth as shown in Fig. 1. The Apollo water landing with three parachutes deployed is shown in the figure. Most of the analytical solutions published in the Apollo reports were based on the von Karman approach, and the space capsule was considered to be a rigid body. However, some Apollo water landing test data showed that for certain impact orientations the bottom-structure's flexibility had a significant effect on the maximum accelerations at the center of gravity (CG) of the space capsule. For these cases, the predictions based on the rigid body spacecraft often underestimated the actual structural responses ${ }^{15,16}$.

The flexible bottom structure of a space capsule is the heat-shield that may be made of stiffened or sandwiched panels with non-uniform thicknesses. Therefore, closed form solutions for a flexible capsule water landing can be difficult to obtain. Since the explicit finite element codes are suitable for modeling complex flexible structures and fluid and structure interactions, it is expected that these codes will be extensively used in simulating the water landing of future space capsules.

This paper provides the results of a preliminary study using LS-DYNA ${ }^{\circledR}$, an explicit nonlinear dynamic finite element code, for simulating a conceptual space capsule water landing. The objective of this preliminary study is to demonstrate that the numerical results obtained are reasonable and can be used to adequately assess design configurations. Several modeling aspects were studied, including determination of an adequate mesh size; comparison of the rigid body results with classical solutions using the von Karman and Wagner approaches; and comparison of the numerical results of a flexible capsule model with published Apollo water impact test data.

The following sections describe the finite element models, computational methods, and numerical results. A representative finite element model of a conceptual space capsule with the surrounding air and water is presented in Section II, the computational methods and approaches are given in Section III, a mesh refinement study is presented in Section IV, and the water landing simulations for both the rigid model and the flexible model are presented in Sections V and VI, respectively. At the end of the paper, concluding remarks are given in Section VII to summarize the findings from this study.

\section{Finite Element Model of a Conceptual Space Capsule}

An early conceptual design of an Apollo-like space capsule for future space exploration is shown in Fig. 2. This design has a maximum diameter of 198 inches and a height of 130 inches. The weight is estimated to be 16,200 lbs. The assumed geometries, CG locations, and mass moments of inertia are also shown in the figure. Two coordinate systems are shown in Figure 2: the one at the cone apex is the global coordinate system and the other at the CG is the local coordinate system. The local coordinate system is attached to and rotates with the vehicle.

A representative finite element (FE) model is shown in Fig. 3. The capsule was discretized with 4-node quad shell elements. The nominal edge length of the capsule shell elements in the impact area is about $3.0 \mathrm{in}$. Coarser meshes were used for the other areas. The air and water were discretized with 8-node solid elements with an ALE multi-material element formulation ${ }^{8}$. In the interaction region shown in Fig. 3, the ALE solid element size is 2.4 in. x 2.4 in. x1.2 in. The air was modeled as a vacuum material. The water domain has 1,218,060 nodes and 1,180,000 elements, and the air domain has 832,341 nodes, and 820,000 elements. The depths of the water and air are 256.8 in. and 168.0 in., respectively.

Note that the mesh of the capsule is immersed in the Eulerian meshes of water and air, but the fluid nodes and structure nodes do not need to be coincident. Figure 3 shows the local coordinate system (fixed on the capsule) that was used for outputting all the CG velocity and acceleration results in this paper. These results can be compared directly with experimental results without coordinate transformations. $V_{V}$ and $V_{H}$ denote the positive directions of vertical and horizontal velocities, respectively. For all analysis cases performed in this study, the vertical landing velocity $\left(\mathrm{V}_{\mathrm{V}}\right)$ is assumed to be $25 \mathrm{ft} / \mathrm{s}$ in the global $\mathrm{X}$ direction and the horizontal velocity $\left(\mathrm{V}_{\mathrm{H}}\right)$ is assumed to be 0 $\mathrm{ft} / \mathrm{s}$ in the in the global negative $Z$ direction.

In this study, air was modeled as a vacuum and water was modeled as a null hydrodynamic material. An equation of state (EOS) was used to compute the water pressure ${ }^{17,18}$. For water in compression, the equation of state used is 


$$
P(\rho)=\mathrm{K}\left(\frac{\rho}{\rho_{o}}-1\right)
$$

where $P$ is the pressure, $\rho$ is the instantaneous density, $\mathrm{K}$ is the initial bulk modulus, and $\rho_{\mathbf{0}}$ is the initial density. The water is assumed to be slightly compressible during landing impact, so the high order terms in Reference 18 are neglected, and the water temperature remains constant.

\section{Computational Methods and Approaches}

The explicit finite element code, LS-DYNA ${ }^{\circledR}$, and an ALE solver were used to analyze the model shown in Fig. 3 , and a penalty method was used to determine the fluid-structure interaction forces (contact forces) between the capsule and the water. This penalty method, allowing fluid to flow around a structure but not through the structure, was used to determine the impact force on a fluid node based on the penetration depth of this node into the structure. The penalty forces were then applied to both the fluid and structure to push the fluid out of the structure.

This study focused on two technical issues: mesh sensitivity and structural flexibility. Mesh refinement studies were performed first to determine an adequate water mesh size. A rigid capsule model was used for the mesh refinement study, so the results could be compared with the closed form solutions. Then, the water landing of a capsule with a flexible bottom structure, referred to as the flexible model, was simulated, and the results were compared with test data in the literature. A flow chart given in Fig. 4 depicts the approaches used for this study.

\section{Mesh Refinement Studies}

To investigate whether a converged solution could be obtained through mesh refinements, FE models with a rigid capsule at a zero-degree pitch angle, using six different water mesh sizes (types) for the impact interaction region, were analyzed. Dimensions of the six different mesh types are listed in Table 1 . The vertical landing velocity $\left(\mathrm{V}_{\mathrm{V}}\right)$ is $25 \mathrm{ft} / \mathrm{s}$, and the horizontal velocity $\left(\mathrm{V}_{\mathrm{H}}\right)$ is $0 \mathrm{ft} / \mathrm{s}$.

Table 1 Solid element dimensions for various mesh types

\begin{tabular}{|l|l|l|l|l|}
\hline \multirow{3}{*}{$\mathrm{W}$} & Mesh Types & L, in & W, in & D, in \\
\cline { 2 - 5 } & $6 \times 6 \times 6$ & 6 & 6 & 6 \\
\cline { 2 - 5 } & $6 \times 6 \times 2.4$ & 6 & 6 & 2.4 \\
\cline { 2 - 5 } \\
\cline { 2 - 5 } & $2.4 \times 2.4 \times 2.4$ & 2.4 & 2.4 & 2.4 \\
\cline { 2 - 5 } & $2.4 \times 2.4 \times 1.2$ & 2.4 & 2.4 & 1.2 \\
\cline { 2 - 5 } & $1.2 \times 1.2 \times 1.2$ & 1.2 & 1.2 & 0.2 \\
\cline { 2 - 5 } & $1.2 \times 1.2 \times 0.6$ & 1.2 & 2 & 0.6 \\
\hline
\end{tabular}

Results for the CG X-velocity and X-acceleration histories are shown in Figs. 5 and 6, respectively. For all the FE models, the bottom of the capsule was discretized with approximate 3 in. $\mathrm{x} 3$ in. quadrilateral elements. In this paper, the negative value of acceleration is the deceleration; and the acceleration and the absolute value of acceleration are used interchangeably depending on the content of the text. Note that the predicted $\mathrm{X}$-acceleration time histories contain high frequency oscillations. These high frequency oscillations were filtered out with a $100 \mathrm{~Hz}$ low-pass filter (FIR100) ${ }^{19}$. The filtered time histories are plotted in Fig. 6. Figure 5 shows that the CG velocity for Mesh Type 6x6x6 decreases much faster than that computed with the other finer mesh models. Comparison of the $\mathrm{X}$-velocity curves for Mesh Types $2.4 \times 2.4 \times 1.2$ and the $1.2 \times 1.2 \times 1.2$ showed very small difference. Figure 6 shows that the maximum X-acceleration at the CG is continuously reduced as the mesh size becomes finer. Comparison of the X-acceleration curves for Mesh Types 2.4x2.4x1.2 and 1.2x1.2x1.2 showed that nearly the same maximum accelerations are obtained. Note the results for Mesh Type 1.2x1.2x 0.6 are nearly the same as the results for Mesh Type 1.2x1.2x1.2 as shown in Figs. 5 and 6. For clarity, there are only a few data points for Mesh Type 1.2x1.2x 0.6 shown in Fig. 5.

Figure 7 contains the CG maximum X-acceleration as a function of the element volume. The mesh refinement, from a size of 2.4 in. $x 2.4$ in. $x 1.2$ in., to 1.2 in. x 1.2 in. x 1.2 in., and then to 1.2 in. x 1.2 in. $x 0.6$ in., yields no appreciable change in the maximum $\mathrm{X}$-accelerations. A refinement of the capsule mesh was also performed in which 
the quadrilateral elements at the bottom of the capsule were refined from approximate 3 in. $\mathrm{x} 3$ in. to 2 in. $\mathrm{x} 2$ in., and no noticeable change in results from the refined model was found as shown in Fig. 7. By examining the plot in Fig. 7, it is clear that a converged solution has been obtained. The analytical solutions ${ }^{22,23}$ using von Karman and Wagner approaches are also shown in Fig. 7 to illustrate that the converged solution is bounded by the two analytical solutions.

\section{Analysis Results for Rigid Capsule Model}

Water landing analyses results for the capsule, assumed to be rigid, with various pitch angles are presented in this section. In these analyses, Mesh Type 2.4x2.4x1.2 was chosen to model the fluid in the interaction region for computational efficiency. The water model is the same as the one shown in Fig. 3. The time step required for the water landing analysis is $6.84 \times 10^{-6} \mathrm{~s}$. A typical analysis time for simulating the first $50 \mathrm{~ms}$ of the water impact took about 28.5 CPU hours on a single $2 \mathrm{GHz}$ Opteron ${ }^{\circledR} 864$ processor.

To illustrate that the capsule position and the water splash (jet flow) can be quite realistically simulated, a snap shot at $0.15 \mathrm{~s}$ after impact of the capsule with a fifteen-degree pitch angle landing is shown in Fig. 8. The effect of jet flow on the impact force prediction was considered in Wagner's solutions ${ }^{2,20}$. For this water landing case, the $\mathrm{X}$ acceleration and the Y-rotational acceleration time histories are plotted in Figs. 9 and 10, respectively. Theses two types of accelerations can be combined for estimating the acceleration at other locations on the vehicle. The accelerations along the $\mathrm{Y}$ and $\mathrm{Z}$ axes are small and not presented in this paper. As shown in Figs. 9 and 10 , the acceleration curves contain numerous high frequency oscillations. During the impact, the wetted surface of the bottom structure grows continuously with time, and the peak pressure is located at the edge of the wetted area ${ }^{13,15}$. In the explicit finite element simulations, the continuous growth of the wetted area introduces a series of discrete impacts, each of which is associated with a spike in the interface force ${ }^{21}$. These intermittent spike forces are considered to be the sources of the oscillatory noise in the acceleration history curves. The high frequency noise was filtered out with a FIR100 19 filter. Filtered data are plotted in Figs. 9 and 10 for the X-acceleration and the Yrotational acceleration, respectively.

Filtered X-acceleration time histories for all pitch angles are plotted in Fig. 11 for comparison. For pitch angles less than or equal to 20 degrees, the maximum accelerations occurred within the first five milliseconds after impact. X-accelerations were small for pitch angles of 30 and 40 degrees. The maximum acceleration as a function of pitch angle is plotted in Fig. 12 which shows that the maximum accelerations for 0, 10, and 15-degree pitch angles are around $15 \mathrm{~g}$ and for 30 and 40-degree pitch angles are below $5 \mathrm{~g}$. There is a large reduction of the maximum Xacceleration when the pitch angles increase from 15 degrees to 30 degrees. A similar trend was found in the Apollo reports reviewed. Note that an entry angle of 27.5 degrees was used for the Apollo space capsule water landing ${ }^{15}$. The analytical solutions ${ }^{22,23}$ using von Karman and Wagner approaches are also plotted in Fig. 12. Since the maximum X-acceleration for the 0-degree pitch angle is bounded by the two analytical solutions, this indicates that the numerical solutions obtained are reasonable.

Y-rotational acceleration histories for all pitch angles are plotted in Fig. 13 for comparison. Since the CG is not located at the capsule's axis of symmetry (see Figure 2 for the CG locations), small negative Y-rotational accelerations were predicated instead of zero for the 0-degree pitch angle. Large Y-rotational accelerations were found for pitch angles of 15 degrees and 20 degrees. For pitch angles above 30 degrees, the Y-rotational accelerations decreased to below $20 \mathrm{rad} / \mathrm{s}^{2}$. The maximum Y-rotational accelerations as a function of pitch angles are plotted in Fig. 14. The maximum Y-rotational acceleration curve reaches its peak about $60 \mathrm{rad} / \mathrm{s}^{2}$ at a pitch angle around 17 degrees.

\section{Analysis Results for Flexible Capsule Model}

For an actual capsule, the bottom structure that impacts the water is expected to be flexible. Experimental data presented in the Apollo reports ${ }^{14,15}$ show that the interaction, between water and a flexible bottom structure (heat shield), can generate much larger impact forces than those of a rigid capsule resulting in larger CG acceleration levels. This increase in acceleration was found for low impact angles. For impact angles greater than 30 degrees, the effect of flexibility is negligible. This part of the study demonstrates that the hydroelastic effect between water and the capsule's flexible bottom structure can be numerically simulated. 
The flexible capsule model shown in Fig. 15 has a flexible bottom portion and a rigid upper portion. The flexible bottom portion, modeled using shell elements, is assumed to be a linear elastic material that has an equivalent Young's modulus of $5.0 \times 10^{6} \mathrm{psi}$, a thickness of $1.0 \mathrm{in}$., and a density of $0.1 \mathrm{lb} / \mathrm{in}^{3}$. The CG location, total mass, and moments of inertia of the flexible model are the same as the previous rigid body model. In the simulations, the capsule landed on water with a vertical velocity of $25 \mathrm{ft} / \mathrm{s}$ and at five pitch angles: $0,10,20,30$, and 40 degrees. This flexible model water landing analysis required a time step about one quarter of the time step required for the rigid body model water landing analysis. A typical analysis for simulating the first $50 \mathrm{~ms}$ of the water impact took about 173.5 CPU hours on a single $2 \mathrm{GHz}$ Opteron ${ }^{\circledR} 864$ processor.

The interaction between the water and the capsule's flexible bottom was investigated to understand the hydroelastic effect on the $\mathrm{X}$-accelerations. For a 0-degree pitch angle landing case, the X-displacement histories for the vehicle CG and for the bottom center point are plotted in Fig. 16. At any instant, the difference between these two curves is the deflection at the center of the bottom surface. A maximum upward deflection (-X direction) of $1.643 \mathrm{in}$. occurred at $14.75 \mathrm{~ms}$. Note, at this instant, the CG and the bottom center point had the same velocity as shown in Fig. 17. After reaching the maximum deflection, the capsule's bottom surface sprang back and created a downward velocity much higher than the initial capsule velocity. This maximum velocity is about $550 \mathrm{in} / \mathrm{s}$ at the bottom center point and is shown in Fig. 17. This high velocity interaction resulted in a large impact force which in turn caused a large deceleration (more than $30 \mathrm{~g}$ ) of the capsule as shown in Fig. 18. Figure 19 shows that the velocity of the flexible model after $14.75 \mathrm{~ms}$ decreases at a much faster rate than that of the rigid model.

The filtered X-acceleration and Y-rotational acceleration histories of the capsule water landing with five different pitch angles are plotted in Figs. 20 and 21. These acceleration histories were compared with those for the rigid capsule shown in Figs. 11 and 13. The maximum acceleration for the flexible model was found to occur at a much later time, around $20 \mathrm{~ms}$, for the same pitch angle landing. Figure 22 plots the maximum acceleration as a function of pitch angle for both the rigid model and the flexible model. The von Karman and Wagner solutions from the Appendix for the 0-degree pitch angles are also shown in the figure. It is apparent that the maximum accelerations for the flexible model at low pitch angles $(<20$ degrees) are much greater than those predicted by the rigid model. However, the difference between the maximum accelerations from the flexible model and the maximum accelerations from the rigid model diminishes as the pitch angle increases. Maximum Y-rotational accelerations from both the rigid model and the flexible model are plotted in Fig. 23. For low pitch angles, much greater Y-rotational accelerations were predicted for the flexible model than for the rigid model. The maximum Yrotational accelerations predicted for the flexible model at pitch angles greater than 20 degrees are nearly the same as those predicted with the rigid model.

\section{Concluding Remarks}

A preliminary study of simulating the water landing of a conceptual space capsule with an explicit finite element code was presented. Tasks performed in this study include conducting a mesh refinement study, comparing the numerical solutions with analytical solutions for the capsule modeled as a rigid body, and investigating the acceleration magnified by the hydroelastic effect for a capsule with a flexible bottom structure.

Mesh refinements were first performed, so an adequate mesh size could be identified for modeling the water and air for the rest of this study. Both the fluid mesh and the capsule mesh were refined to investigate whether a converged solution could be obtained. Since the change of the solutions was found to be insignificant when the meshes were sufficiently refined, this clearly indicates that a converged solution has been obtained.

Analyses were performed for the rigid capsule model entering the water at various pitch angles. The maximum acceleration is about $15 \mathrm{~g}$ for pitch angles less than 15 degrees and less than $5 \mathrm{~g}$ for pitch angles above 30 degrees. Also, it was shown that the maximum X-acceleration for the 0-degree pitch angle case is bounded by the closed form solutions based on the von Karman and Wagner approaches. This indicates that the analyses performed can produce satisfactory results to use in design studies.

Water landing analyses of the flexible capsule model with various pitch angles were performed. The maximum $\mathrm{X}$-accelerations for low pitch angles obtained from the flexible model are significantly greater than the rigid body solutions, nearly twice as large for the 0 -degree pitch angle case. Furthermore, the fluid-structure interaction process during the impact was examined, and it was found that the bottom structure deformed upward at the initial impact. 
Next, the center portion of the bottom surface moved downward to re-impact the water at a speed much greater than the initial impact velocity. The maximum X-acceleration occurred during this re-contact process. The maximum Xaccelerations for pitch angles above 20 degrees predicted by both the flexible model and the rigid model are nearly the same. Thus, for large pitch angles, the water landing solutions from the rigid model may be sufficient for the conceptual design. These findings from the flexible bottom model qualitatively agree with the experimental data of Apollo capsules with a heat shield attached.

In summary, this preliminary study demonstrates that the explicit nonlinear dynamic finite element method is suitable for simulating the water landing of space capsules. By using an adequate water mesh size and accurately modeling the flexibility of the bottom structure, satisfactory numerical results can be obtained.

\section{Appendix: Closed Form Solutions from von Karman and Wagner Approaches}

For a space capsule that has a spherical bottom and is assumed to be rigid, closed form solutions based on the von Karman ${ }^{1}$ and Wagner ${ }^{2}$ approaches are available for correlating with the results from the explicit finite element analyses. The von Karman approach is based on the momentum theorem and using an added virtual mass. The penetration depth is determined without considering the splash-up of water level. The Wagner approach uses rigorous fluid dynamic formulations and considers the effect of the splash-up water level on the impact force.

Analytical solutions for a spherical bottom body impacting with water using the von Karmen method, presented in Ref. 22, are summarized here. The magnitude of the virtual mass for a spherical bottom body is

$$
m=\frac{4}{3} \rho b^{3 / 2}(2 R-b)^{3 / 2}
$$

where $m$ is the virtual mass, $\rho$ is the mass density of water, $b$ is the water depth, and $R$ is the radius of the spherical bottom. The instantaneous velocity, $V$, of the center of gravity of the rigid body is

$$
V=\frac{d b}{d t}=V_{0}(1+m g / W)^{-1}
$$

where $t$ is time after impact, $V_{o}$ is the initial velocity, $g$ is the gravitational constant, and $W$ is the weight of the rigid body. By substituting Eq. A1 into Eq. A2, the instantaneous velocity can be rewritten as

$$
V=\frac{V_{0}}{1+\frac{8 \rho g R^{3}}{3 W}\left(\frac{b}{R}\right)^{3 / 2}}
$$

The overall acceleration, $a$, can be written as

$$
a=\frac{d^{2} b}{d t^{2}}=-\left[\frac{3 \times 2^{1 / 2}\left(\frac{3 W}{4 \pi \rho g R^{3}}\right)^{2}\left(\frac{V_{0}^{2}}{g R}\right)\left(\frac{b}{R}\right)^{1 / 2}}{\pi\left(\left(\frac{3 W}{4 \pi \rho g R^{3}}\right)+2^{3 / 2} \pi^{-1}\left(\frac{b}{R}\right)^{3 / 2}\right)^{3}}\right] g
$$

Assuming $b / R<<1$, the maximum acceleration can be found as

$$
a_{\max }=-\frac{256}{243}\left(\frac{4 \rho g R^{3}}{3 W}\right)^{2 / 3}\left(\frac{V_{0}^{2}}{R}\right)
$$

with the impact time at 


$$
t_{\text {max }}=\frac{21}{160}\left(\frac{3 W}{4 \rho g R^{3}}\right)^{2 / 3} \frac{R}{V_{0}}
$$

and the penetration depth at

$$
b_{\max }=\frac{1}{8}\left(\frac{3 W}{4 \rho g R^{3}}\right)^{2 / 3} R
$$

In the von Karman approach, the rise of water due to the splash up is not considered. The effect of splash up was considered by Wagner and found to have significant effect on the impact force. Recently, Miloh ${ }^{23}$ used a semiWagner approach to determine the slamming coefficient, a non-dimensional parameter that is defined as

$$
C_{s}(b / R)=\frac{2 F}{\rho \pi R^{2} V_{o}^{2}}
$$

where $F$ is the impact force. Base on his analytical derivations, Miloh proposed that

$$
C_{s}\left(\frac{b}{R}\right)=5.50\left(\frac{b}{R}\right)^{1 / 2}-4.19\left(\frac{b}{R}\right)-4.26\left(\frac{b}{R}\right)^{3 / 2}
$$

is suitable for initial stage slamming. The maximum acceleration can be estimated as

$$
a_{\max }^{*}=\frac{g}{2 W} C_{s}\left(b_{\max } / R\right) \rho \pi R^{2} V_{o}^{2}
$$

\section{Acknowledgments}

The authors would like to gratefully acknowledge the valuable discussions and support from Drs. Edwin L. Fasanella and Lucas G. Horta, Messrs. James M. Corliss and Brian H. Mason, and other colleagues.

\section{References}

${ }^{1}$ von Karman, T., The Impact of Seaplane Floats during Landing, Technical Note 321 NACA, Washington, D.C., 1929.

${ }^{2}$ Wagner, H., “Über Stoss und Gleitvorgänge an der Oberfläche von Flüssigkeiten,” ZAMM Vol. 12, 1932, pp. $193-215$.

${ }^{3}$ Seddon, C. M., and Moatamedi, M., "Review of Water Entry with Applications to Aerospace Structures," International Journal of Impact Engineering, Vol. 32, No. 7, 2006, pp. 1045-1067.

${ }^{4}$ Lucy, L. B., “Numerical Approach to Testing the Fission Hypothesis,” The Astronomical Journal, Vol. 82, 1977, pp. 10131024.

${ }^{5}$ Gingold, R. A., and Monaghan, J. J., "Smoothed Particle Hydrodynamics: Theory and Application to Non-Spherical Stars," Monthly Notices of the Royal Astronomical Society, Vol. 181, 1977, pp. 375-389.

${ }^{6}$ Belytschko, T., Liu, W. K., and Moran, B., Nonlinear Finite Elements for Continua and Structures, John Wiley \& Sons, Inc., West Sussex, England, ISBN 0-471-98773-5, 2000.

${ }^{7}$ Benson, D. J., "Computational Methods in Lagrangian and Eulerian Hydrocodes," Computer Methods in Applied Mechanics and Engineering, Vol. 99, 1992, pp. 235-394.

${ }^{8}$ Hallquist, J. O., LS-DYNA Theoretical Manual, Livermore Software Technology Corporation, Livermore, California, March 2006.

${ }^{9}$ MSC.Dytran User's Manual Version 4.0, The MacNeal-Schwendler Corporation, Los Angles, CA, November 1997.

${ }^{10}$ Fasanella, E. L., Jackson, K. E., Sparks, C. E., and Sareen, A. K., "Water Impact Test and Simulation of a Composite Energy Absorbing Fuselage Section,” Presented at the American Helicopter Society $59^{\text {th }}$ annual Forum, Phoenix, AZ, May 6-8, 2003.

${ }^{11}$ Brooks, J. R., and Anderson, L. A., “Dynamics of a Space Module Impacting Water,” Journal of Spacecraft and Rockets, Vol. 31, No. 3, May-June 1994.

${ }^{12}$ Stenius, I., Rosen, A., and Kuttenleuler, J., “Explicit FE-modeling of Fluid-Structure Interaction in Hull-Water Impacts,” International Shipbuilding Progress, Vol. 53, No. 2, 2006, pp. 103-121.

${ }^{13}$ Stubbs, S. M., Dynamic Model Investigation of Water Pressures and Accelerations Encountered During Landings of the Apollo Spacecraft, NASA TN D-3980, September 1967. 
${ }^{14}$ Stubbs, S. M., and Hathaway, M. E., Effects of Bottom-Structure Flexibility on Water Landing Loads of Apollo Spacecraft Models, NASA TN D-5108, March 1969.

${ }^{15}$ Benson, H. E., "Water Impact of the Apollo Spacecraft,” Journal of Spacecraft and Rockets, Vol. 3, No. 8, August 1966, pp. $1282-1284$.

${ }^{16}$ Wilkinson, J. P. D., Cappelli, A. P., and Salzman, R. N., "Hydroelastic Interaction of Shells of Revolution during Water Impact," AIAA Journal, Vol. 6, No. 5, 1967, pp. 792-797.

${ }^{17}$ Legay, A., Chessa, J., and Belytschko, T., “An Eulerian-Lagrangian Method for Fluid-Structure Interaction Based on Level Set,” Computer Methods in Applied Mechanics and Engineering, Vol. 195, 2006, pp. 2070-2087.

${ }^{18}$ Shin, Y. S., Lee, M., Lam, K. Y., and Yeo, K. S., "Modeling Mitigation Effects of Watershield on Shock Waves," Shock and Vibration, Vol. 5, No. 4, 1998, pp. 225-234.

${ }^{19}$ Federal motor vehicle safety standards No. 214: Side impact protection, Federal Register, Vol. 55, 1990, pp. 4572245780.

${ }^{20}$ Zhao, R., and Faltinsen, O., “Water Entry of Two-Dimensional Bodies,” Journal of Fluid Mechanics, Vol. 246, 1993, pp. 593-612.

${ }^{21}$ Belytschko, T., and Mullen, R., “Two-Dimensional Fluid-Structure Impact Computations with Regularization,” Computer Methods in Applied Mechanics and Engineering, Vol. 27, No. 2, 1981, pp. 139-154.

${ }^{22}$ Hirano, Y., and Miura, K., "Water Impact Accelerations of Axially Symmetric Bodies,” Journal of Spacecraft and Rockets, Vol. 7, No. 6, 1970, pp. 762-764.

${ }^{23}$ Miloh, T., "On the Initial Stage Slamming of a Rigid Sphere in a Vertical Water Entry,” Applied Ocean Research, 1991, Vol. 13, No. 1, pp. 43-48.

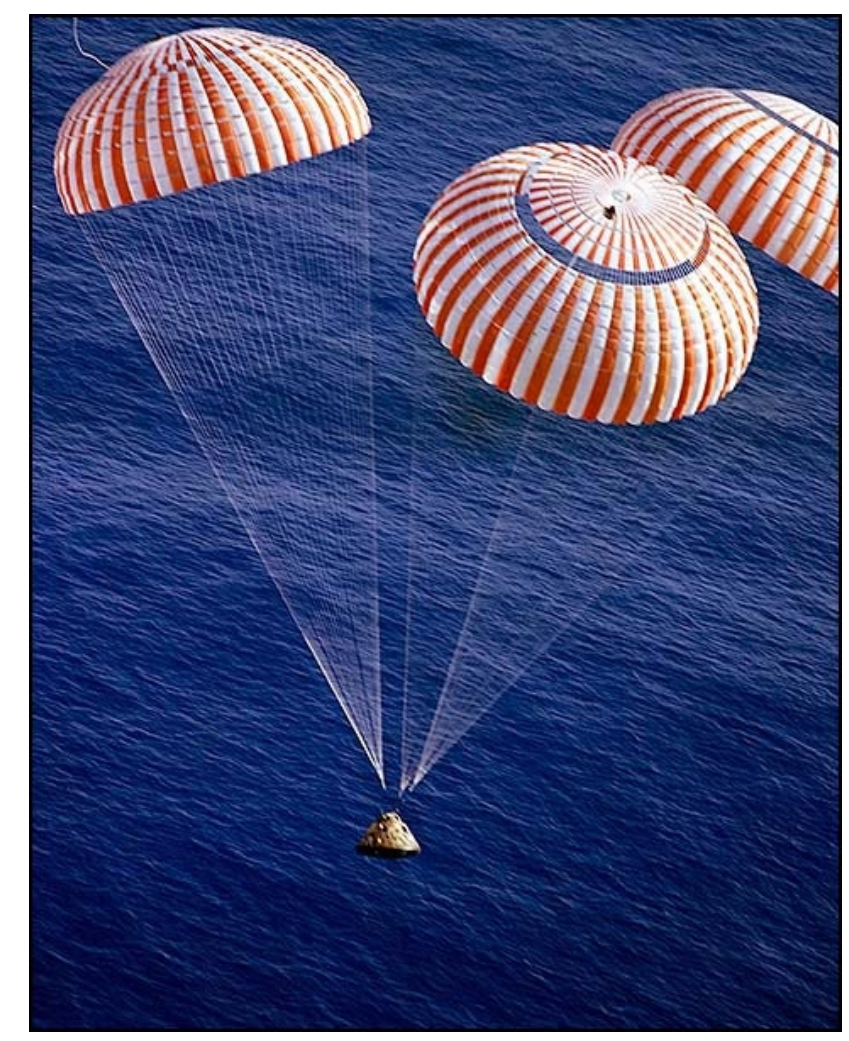

Fig. 1 Apollo water landing with three parachutes deployed. 


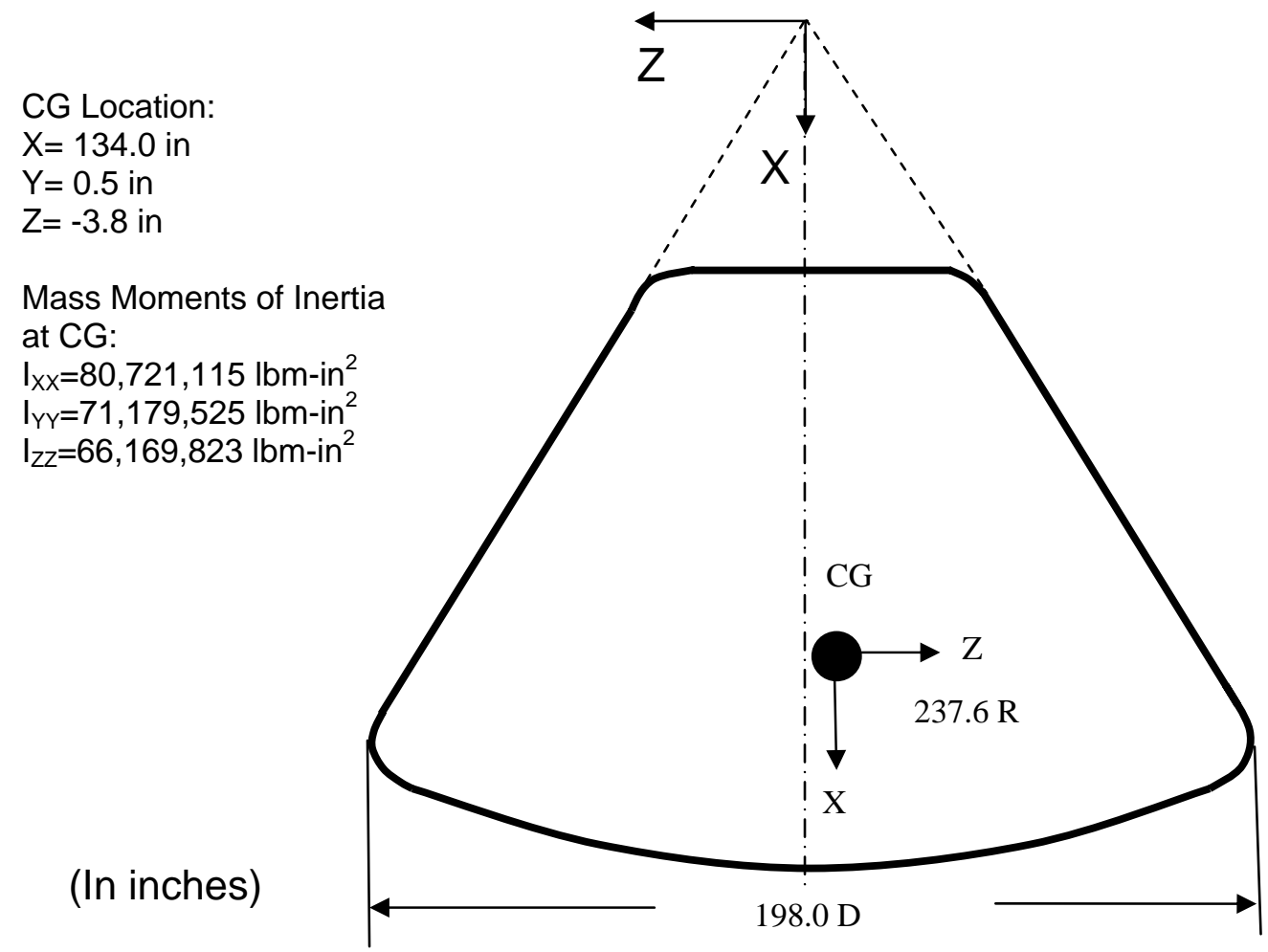

Fig. 2 Dimensions of the space capsule, the global coordinate system at the apex, and a local coordinate system at CG.

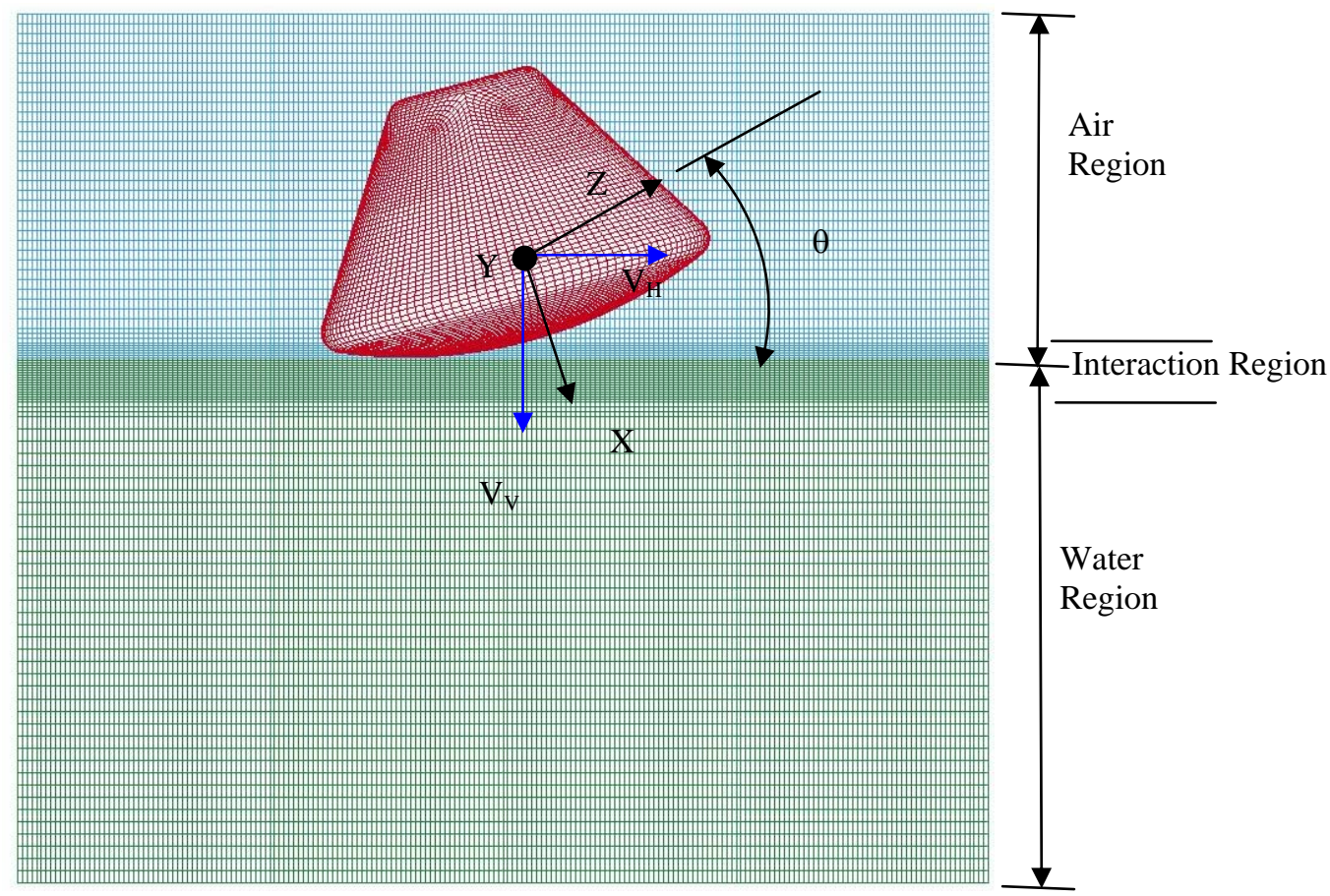

Fig. 3 Finite element model and the local coordinate system at $C G$ with positive vertical velocity $\left(V_{V}\right)$ and positive horizontal velocity $\left(V_{H}\right)$ normal and parallel to the water surface, respectively. 


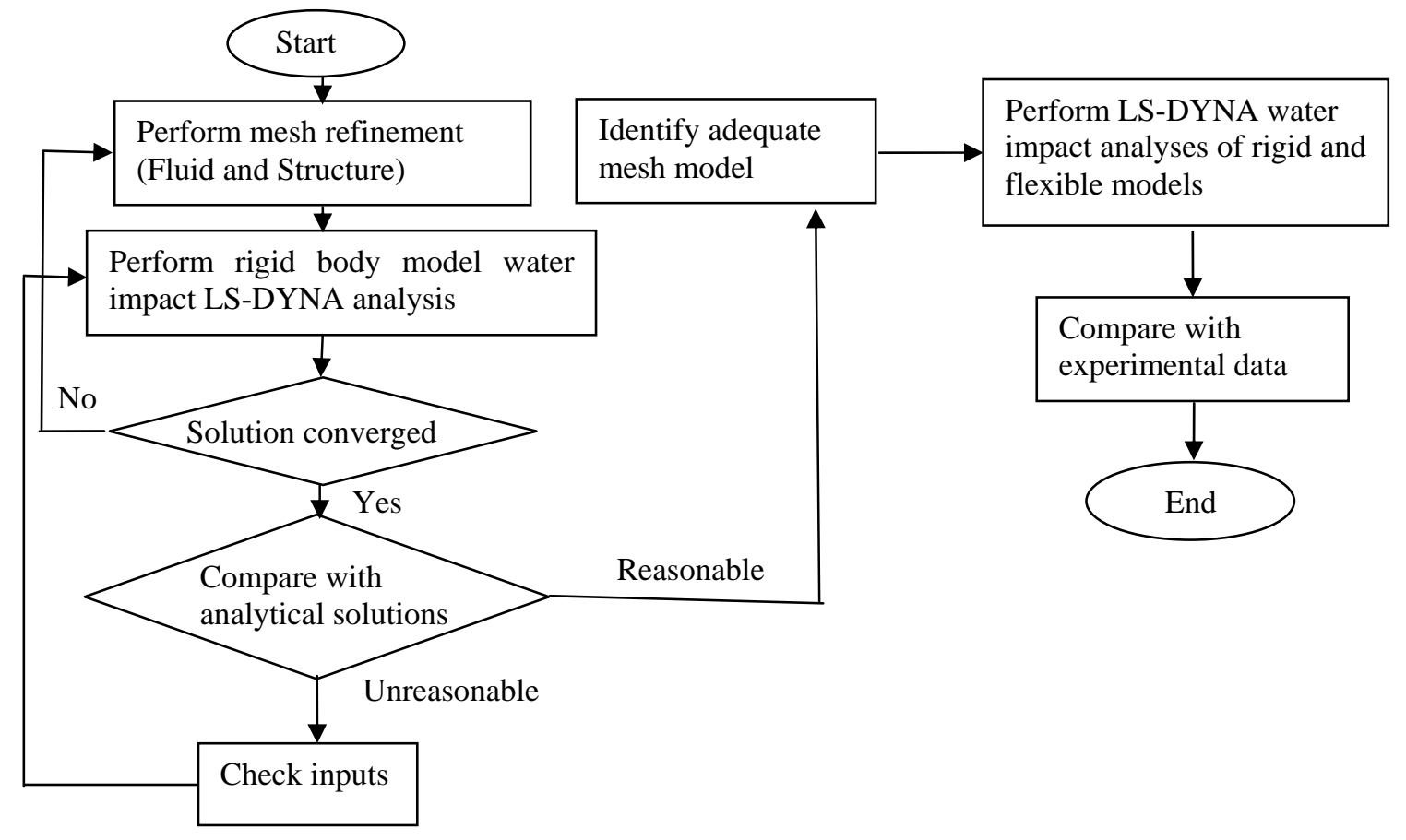

Fig. 4 Flow chart showing the approaches used for this study.

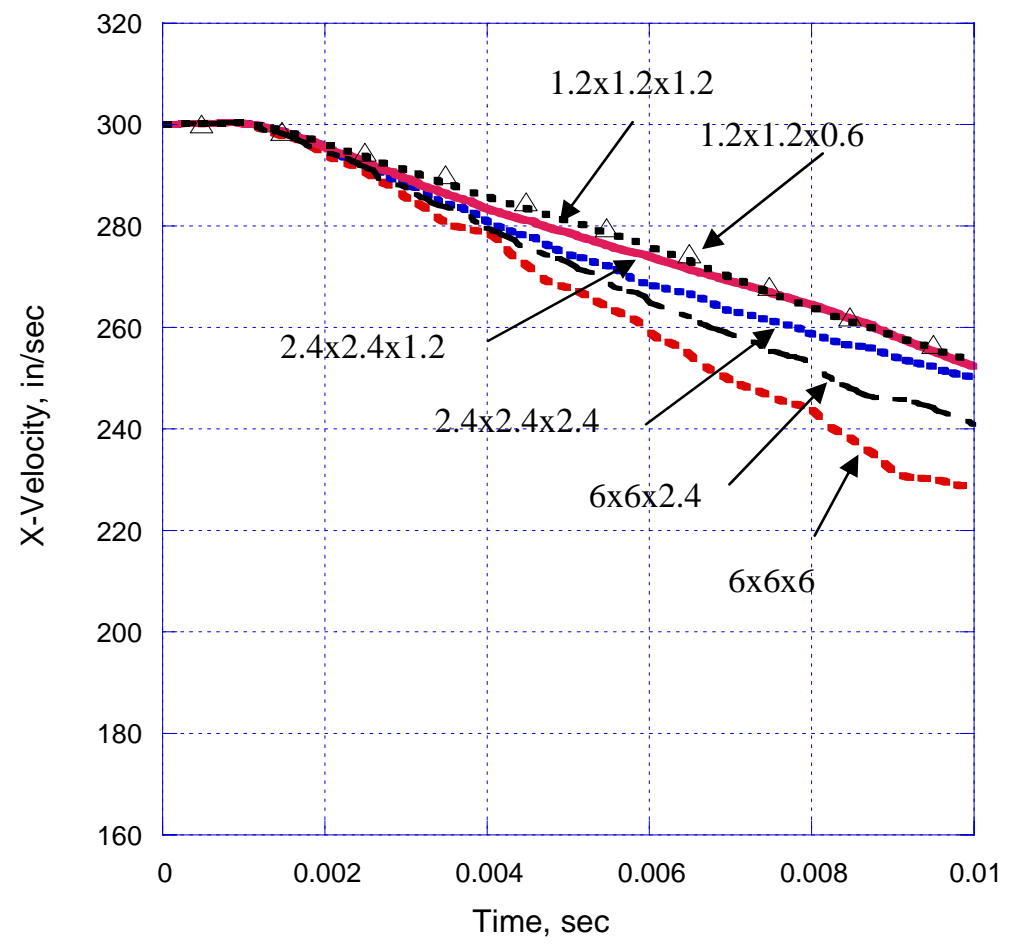

Fig. 5 CG X-velocity histories for various size meshes.

American Institute of Aeronautics and Astronautics 


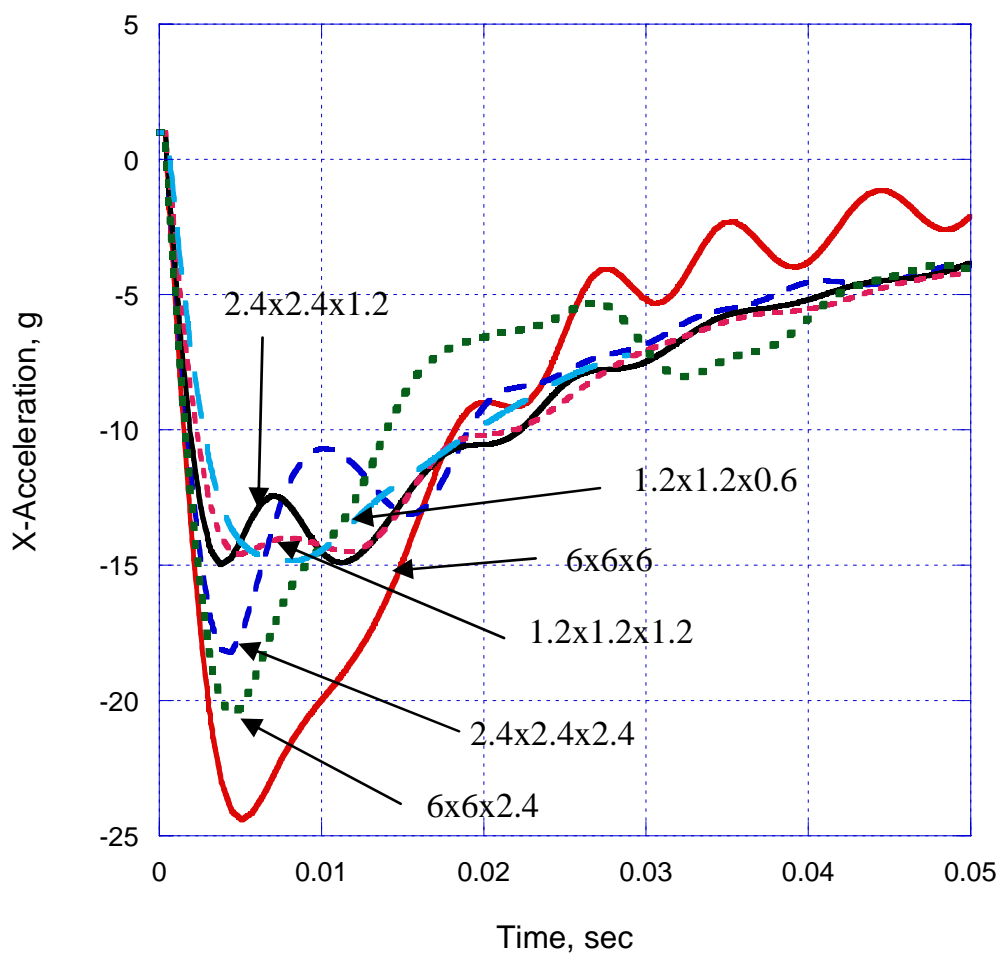

Fig. 6 Filtered $X$-acceleration histories for various size meshes.

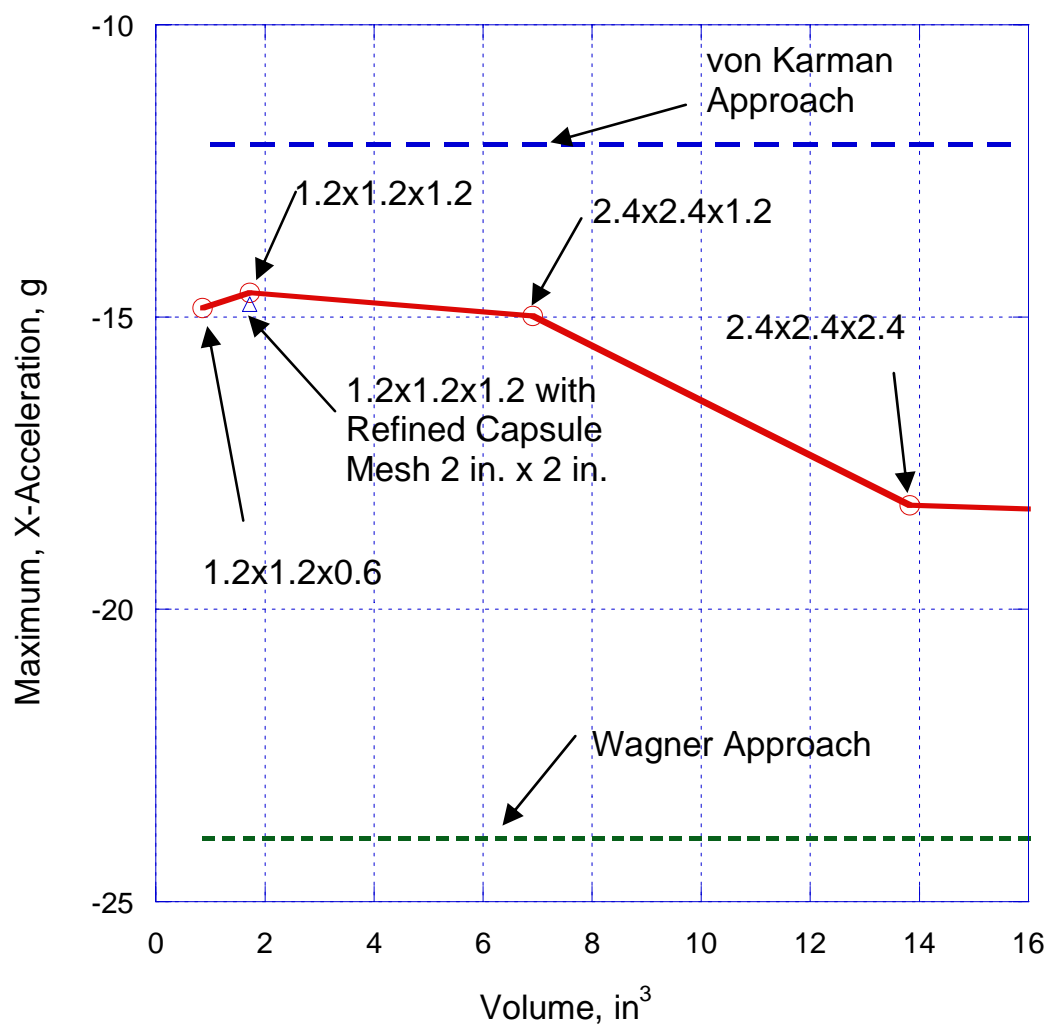

Fig. 7 Maximum $\mathrm{X}$-acceleration converged as the water mesh size refined. 


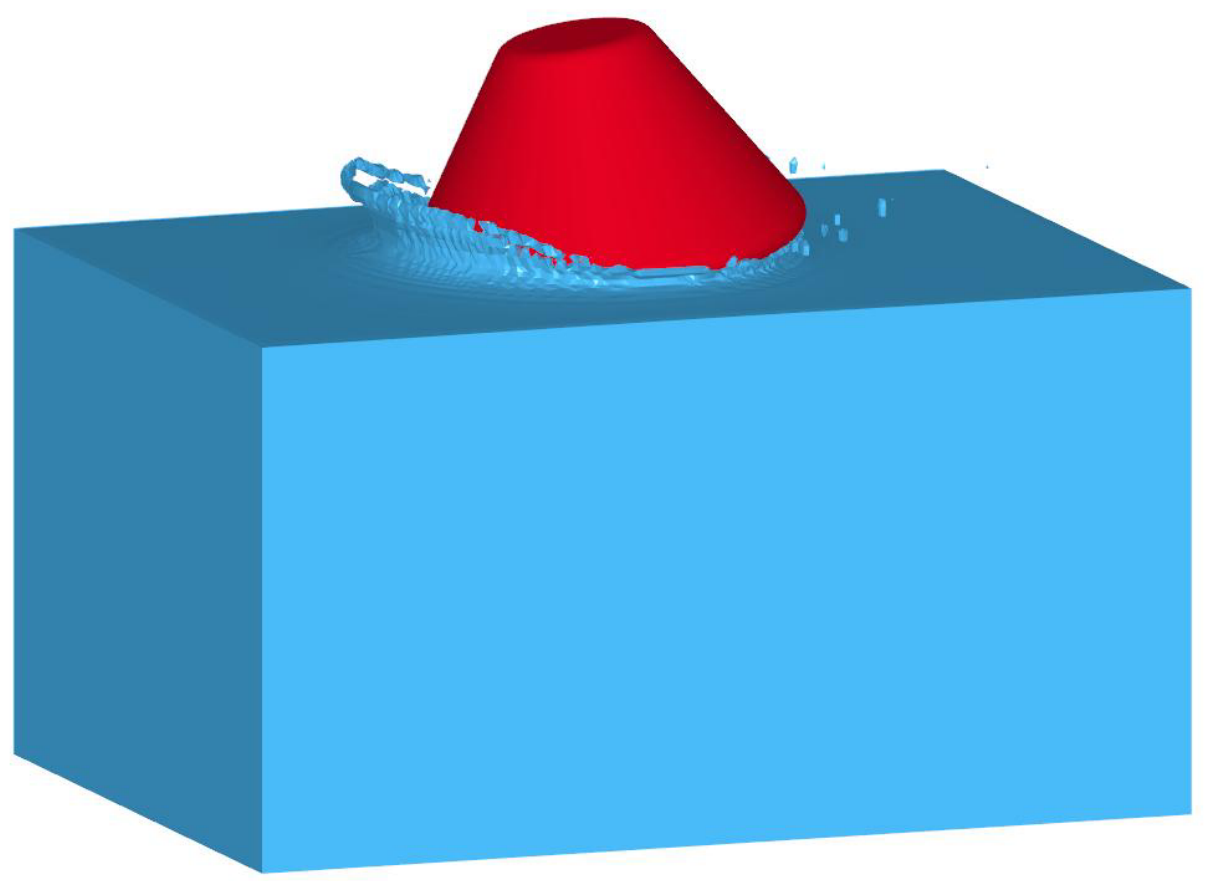

Fig. 8 Capsule water landing with 15-deg pitch angle showing the splash of water.

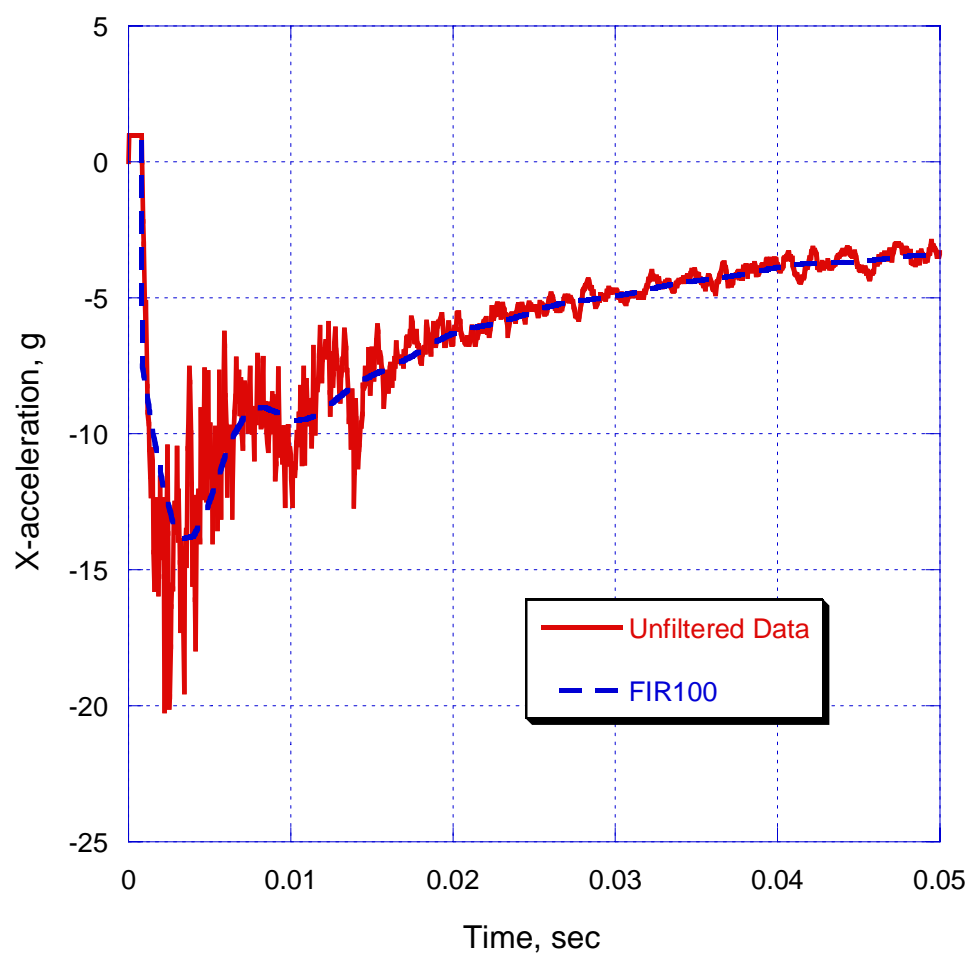

Fig. 9 Unfiltered and filtered $X$-acceleration histories for the water landing of the rigid capsule with a 15deg pitch angle. 


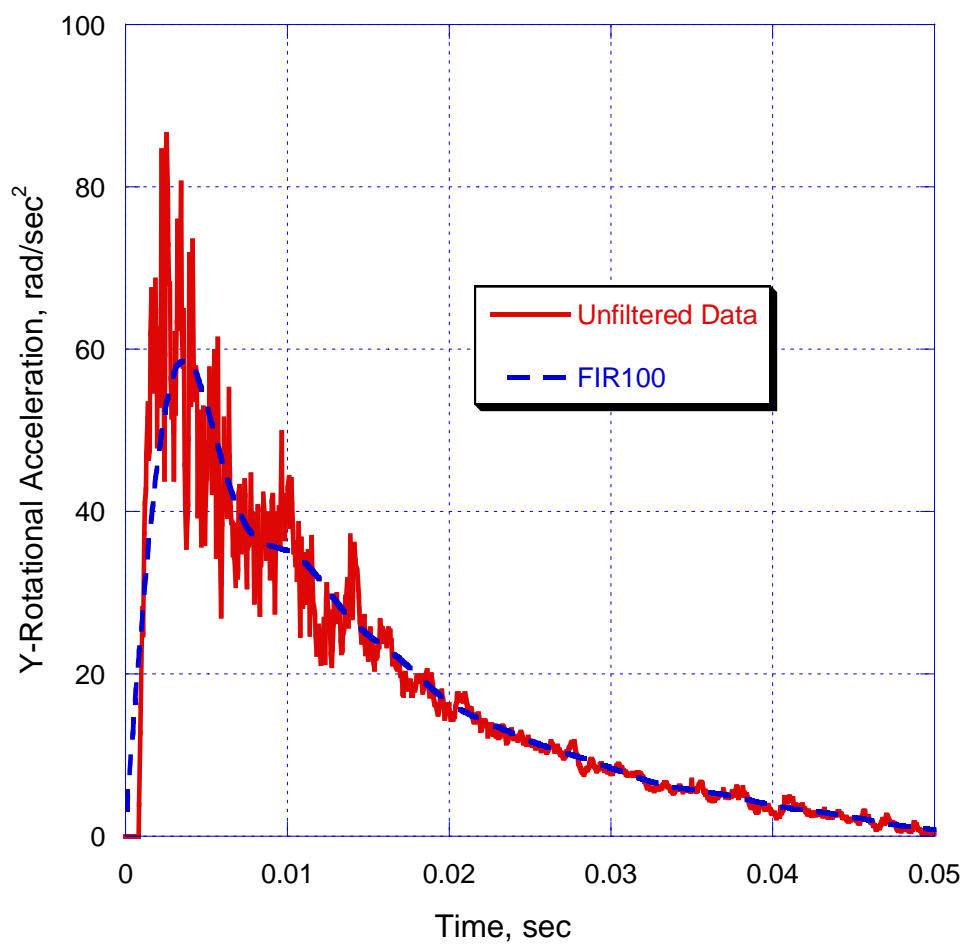

Fig. 10 Unfiltered and filtered Y-rotational acceleration histories for the water landing of the rigid capsule with a 15-deg pitch angle.

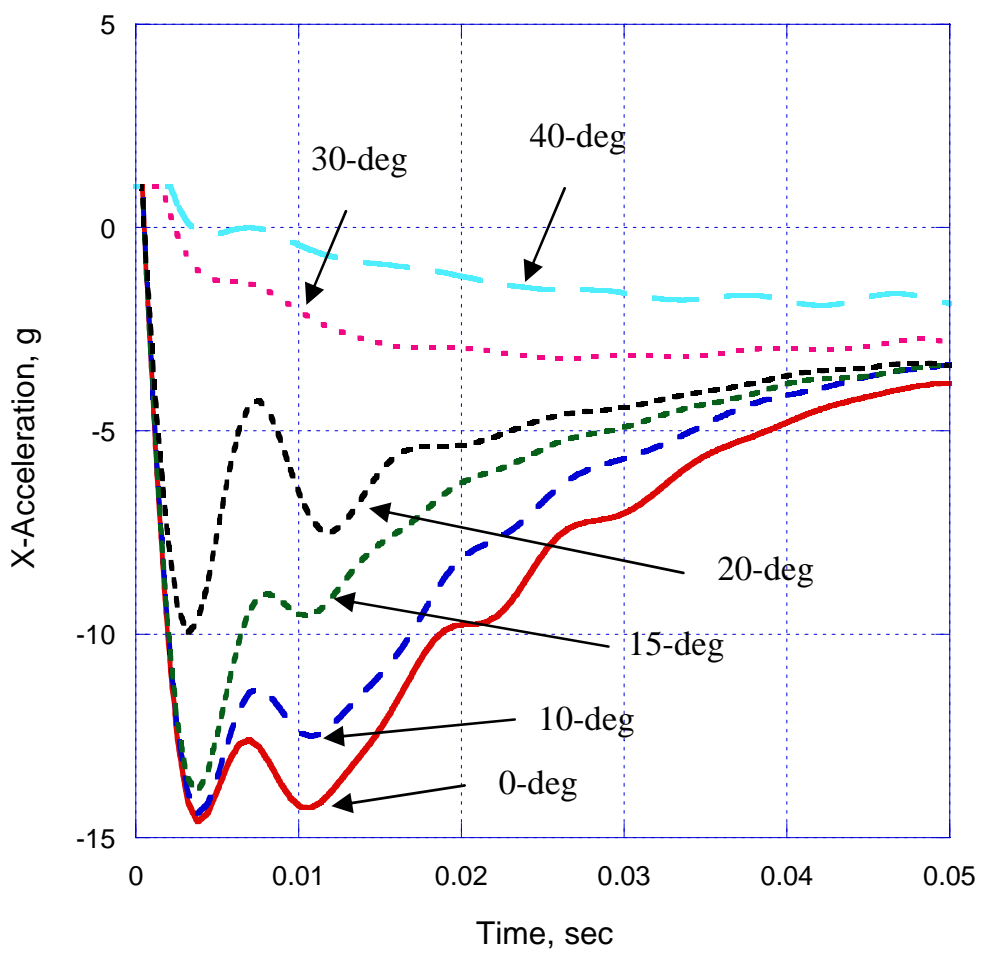

Fig. 11 Filtered $\mathrm{X}$-acceleration histories for the water landing of the rigid capsule with various pitch angles. 


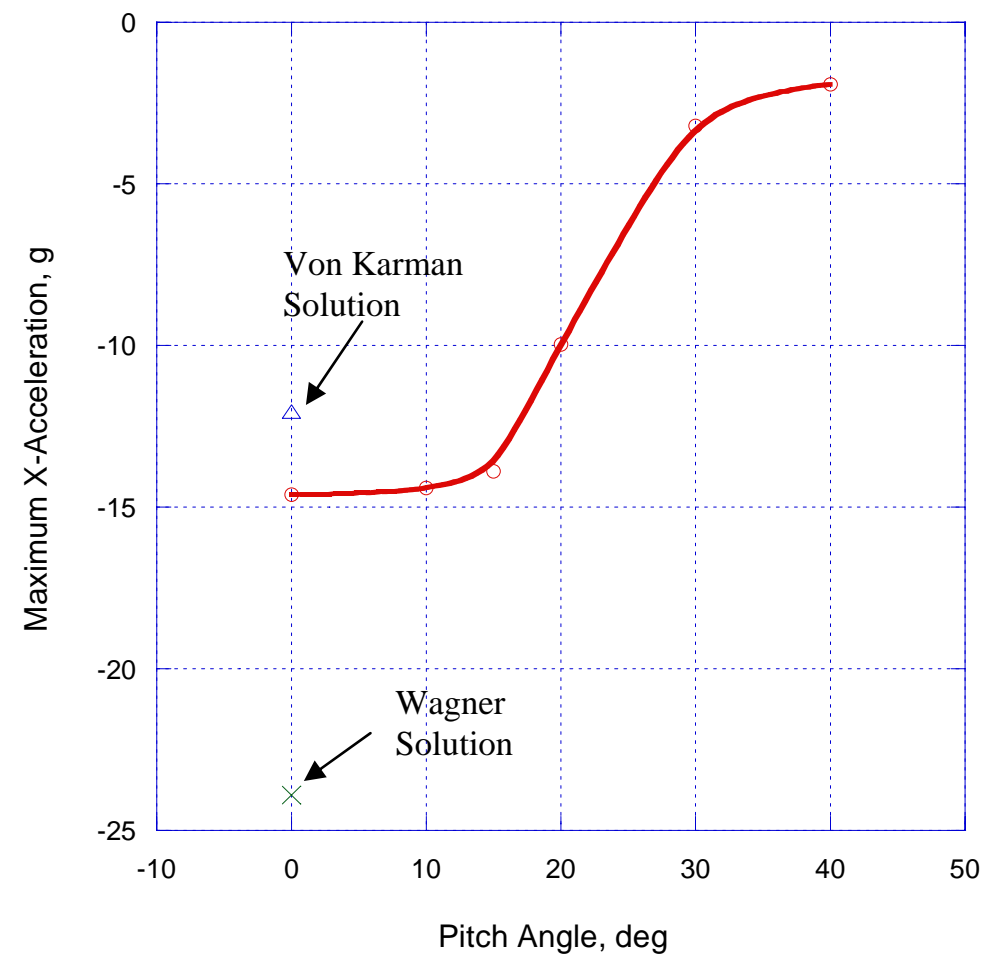

Fig. 12 Maximum $\mathrm{X}$-acceleration as a function of pitch angle for the water landing of the rigid capsule with various pitch angles.

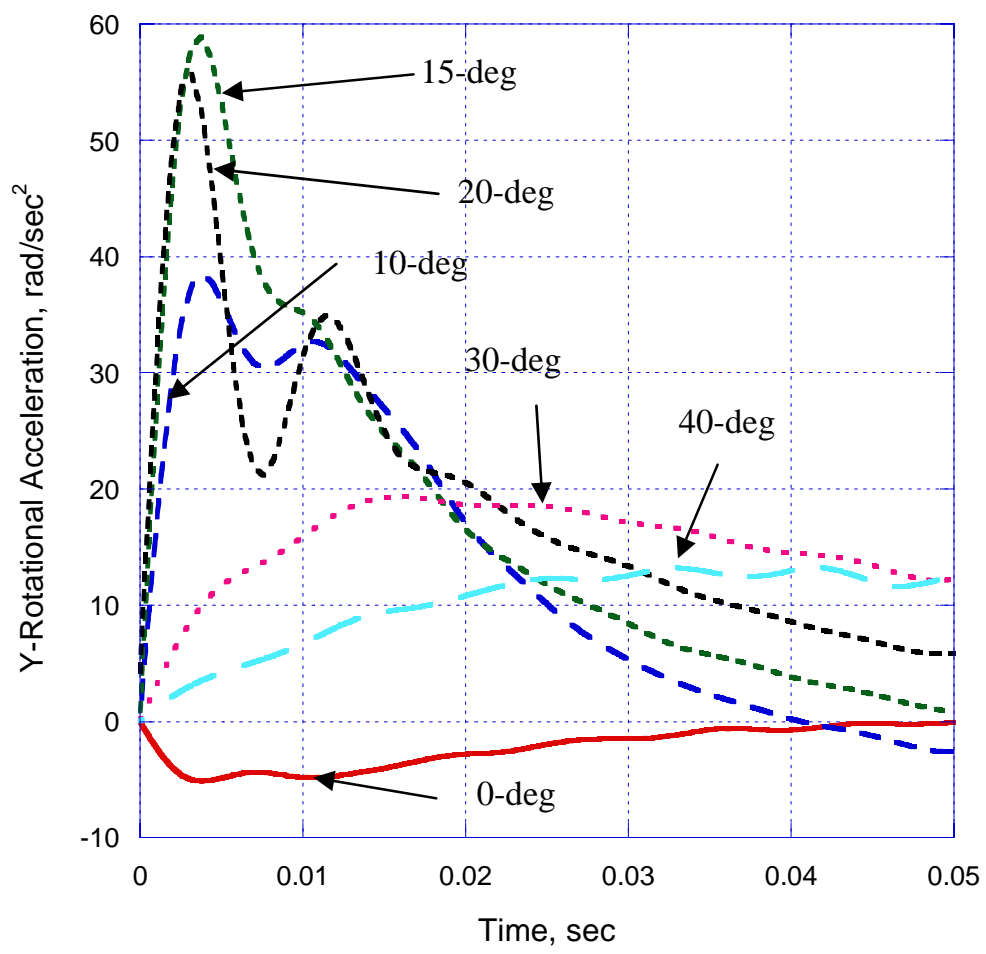

Fig. 13 Filtered Y-rotational acceleration histories for the water landing of the rigid capsule with various pitch angles. 


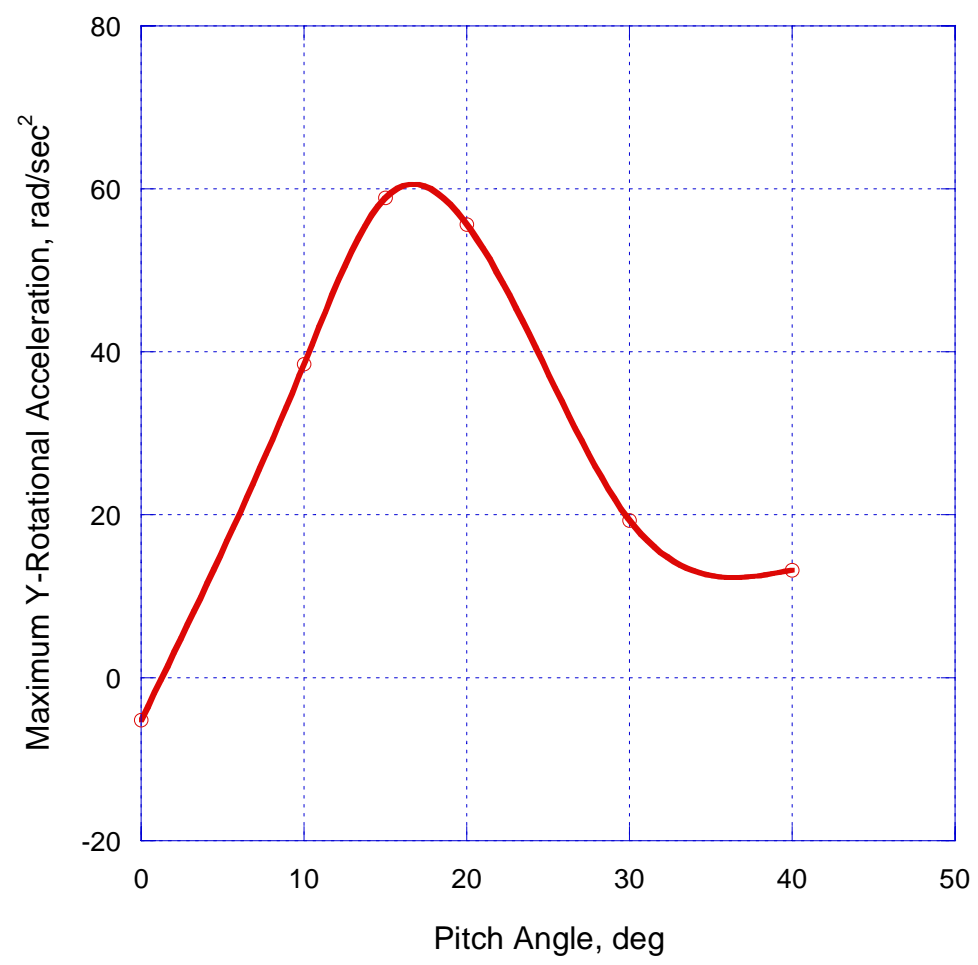

Fig. 14 Maximum Y-rotational acceleration as a function of pitch angle for the water landing of the rigid capsule.

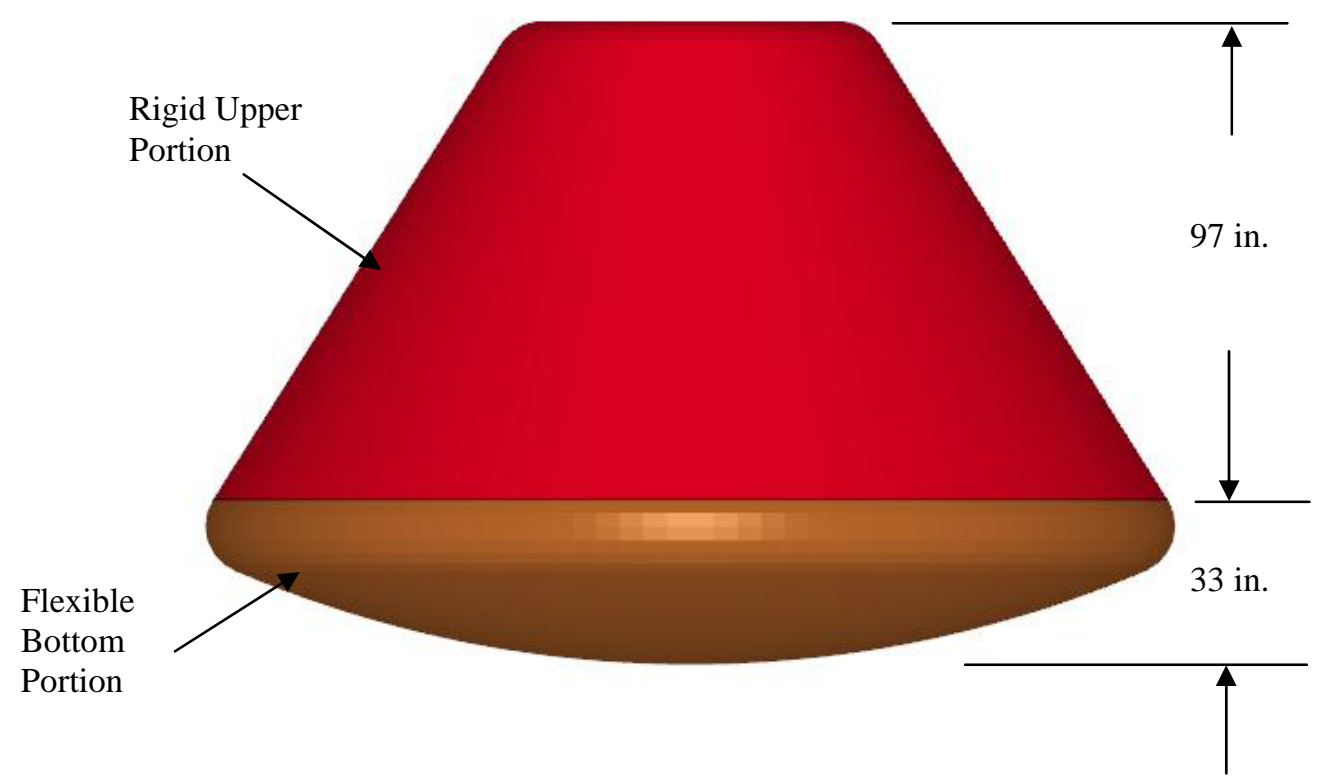

Fig. 15 The flexible capsule model containing a flexible bottom portion and a rigid upper portion. 


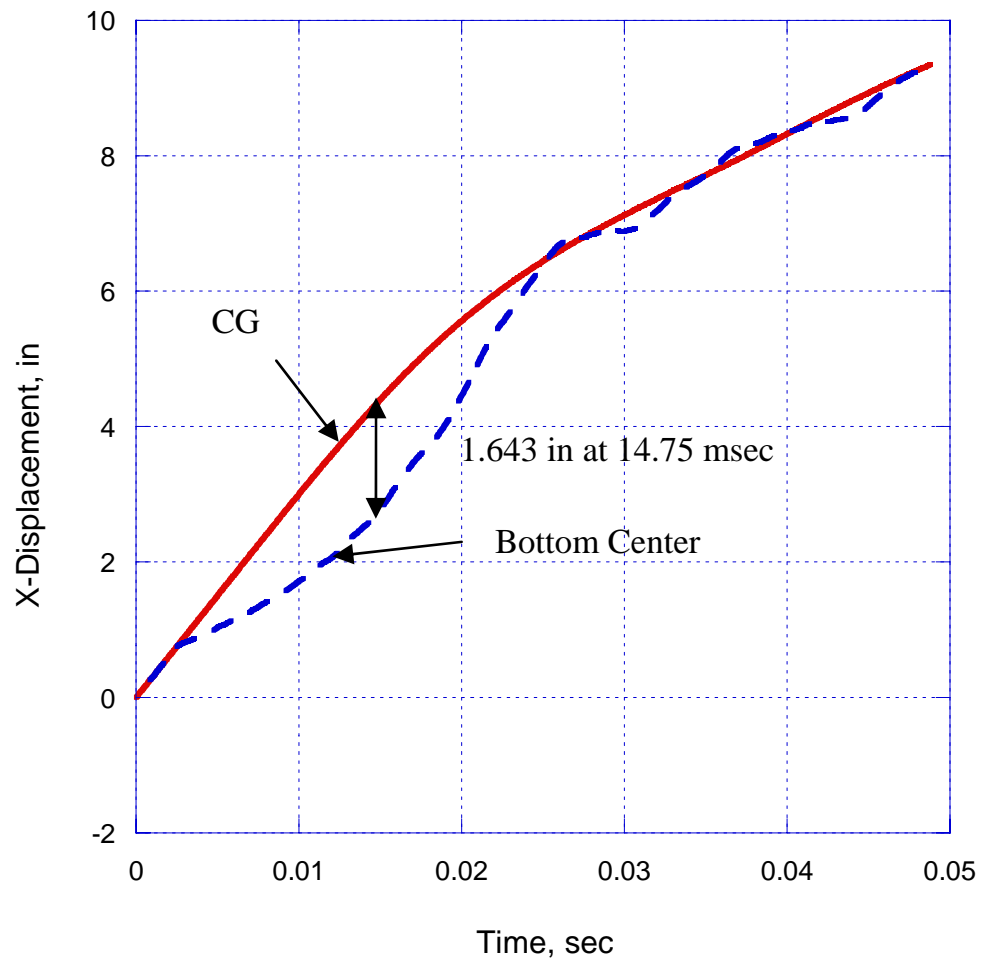

Fig. $16 \mathrm{X}$-displacement histories at the CG and the center of the bottom structure for 0-deg pitch angle landing.

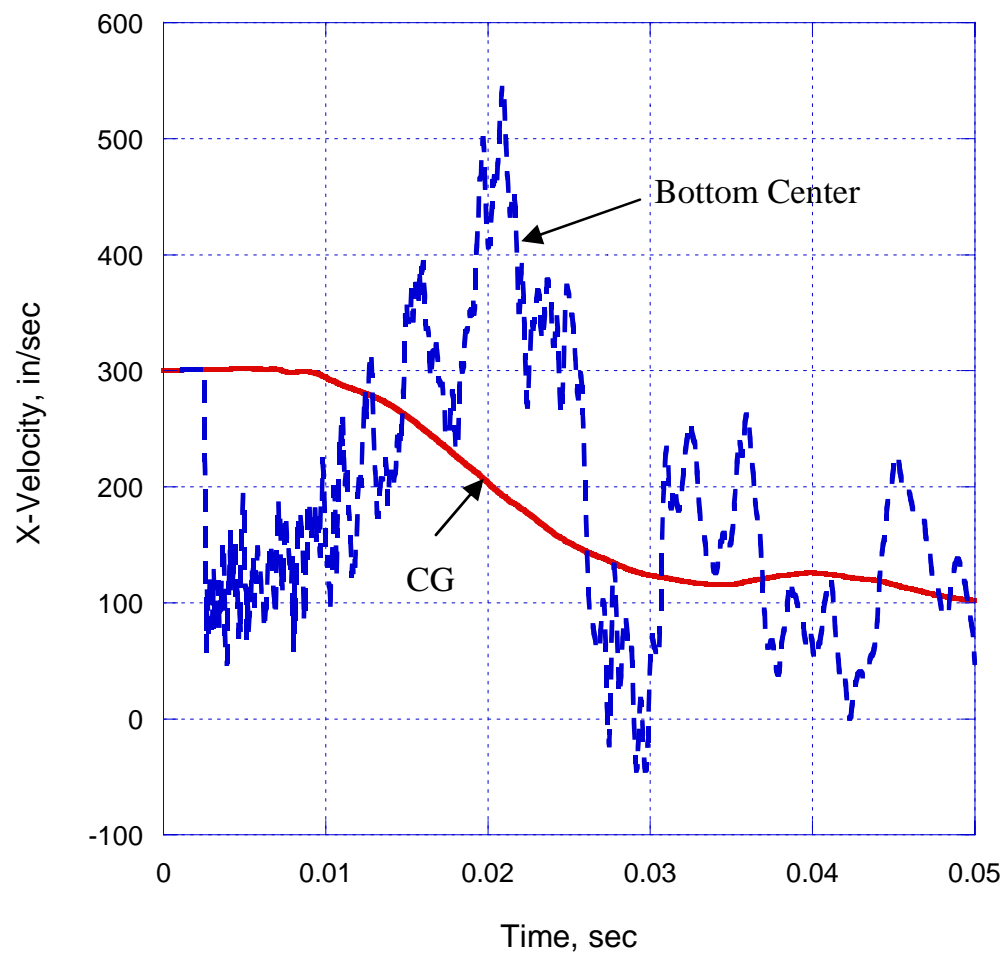

Fig. 17 Velocity histories at CG and the center of the bottom structure for 0-deg pitch angle landing. 


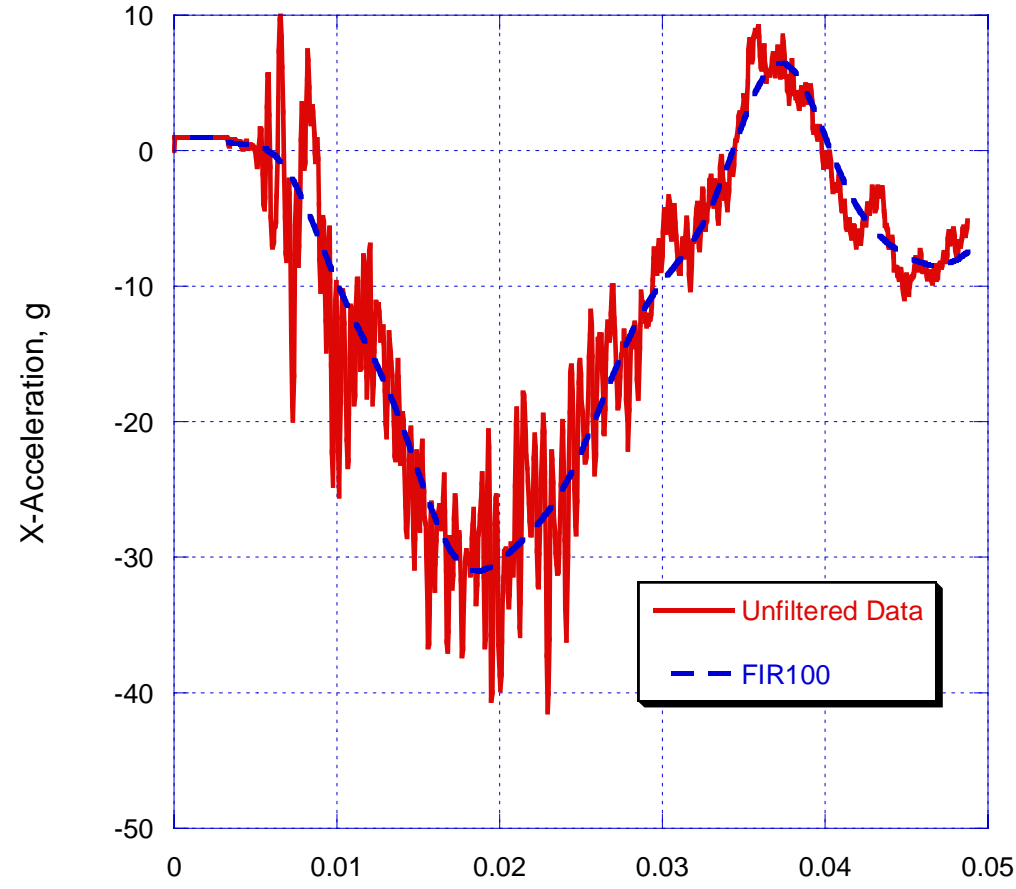

Time, sec

Fig. 18 Filtered and unfiltered $X$-acceleration histories for the water landing of the flexible model with 0 deg pitch angle.

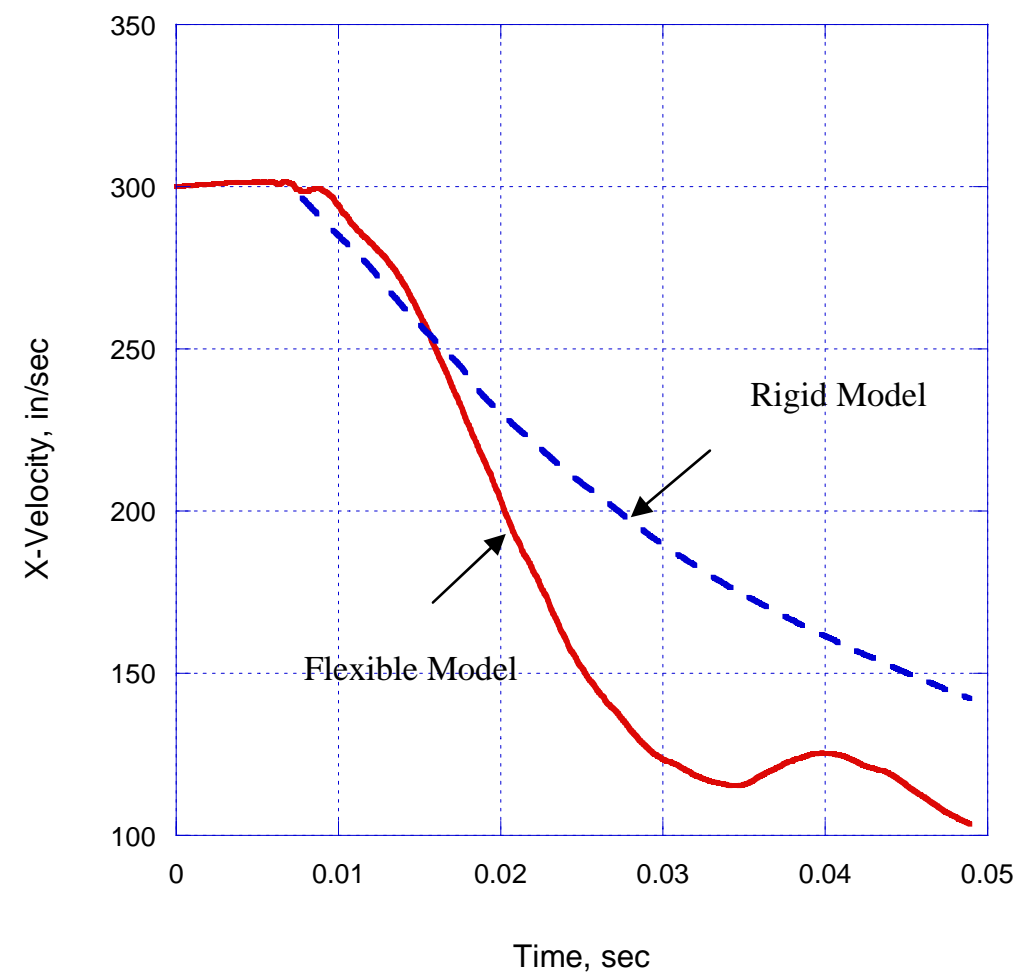

Fig. 19 Velocity histories for the rigid capsule and the flexible capsule models with a 0-deg pitch angle. 


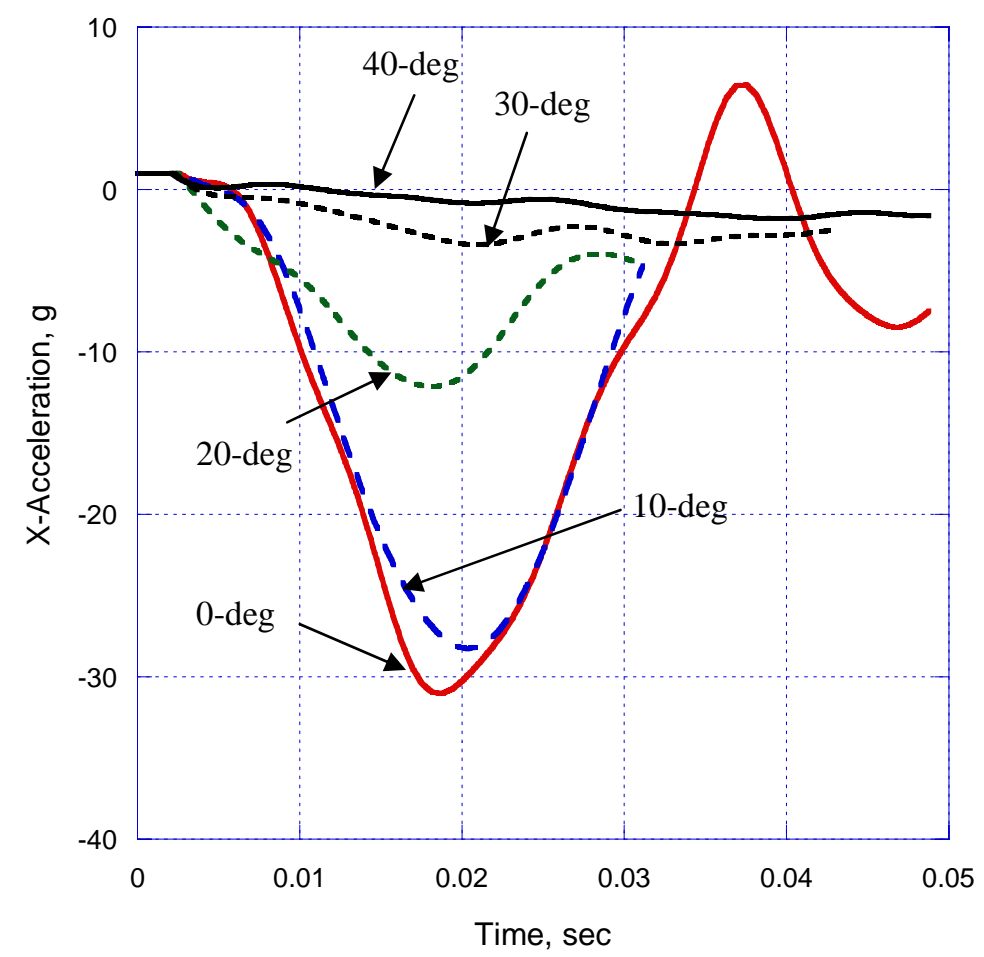

Fig. 20 Filtered $\mathrm{X}$-acceleration histories for the flexible model with various pitch angles.

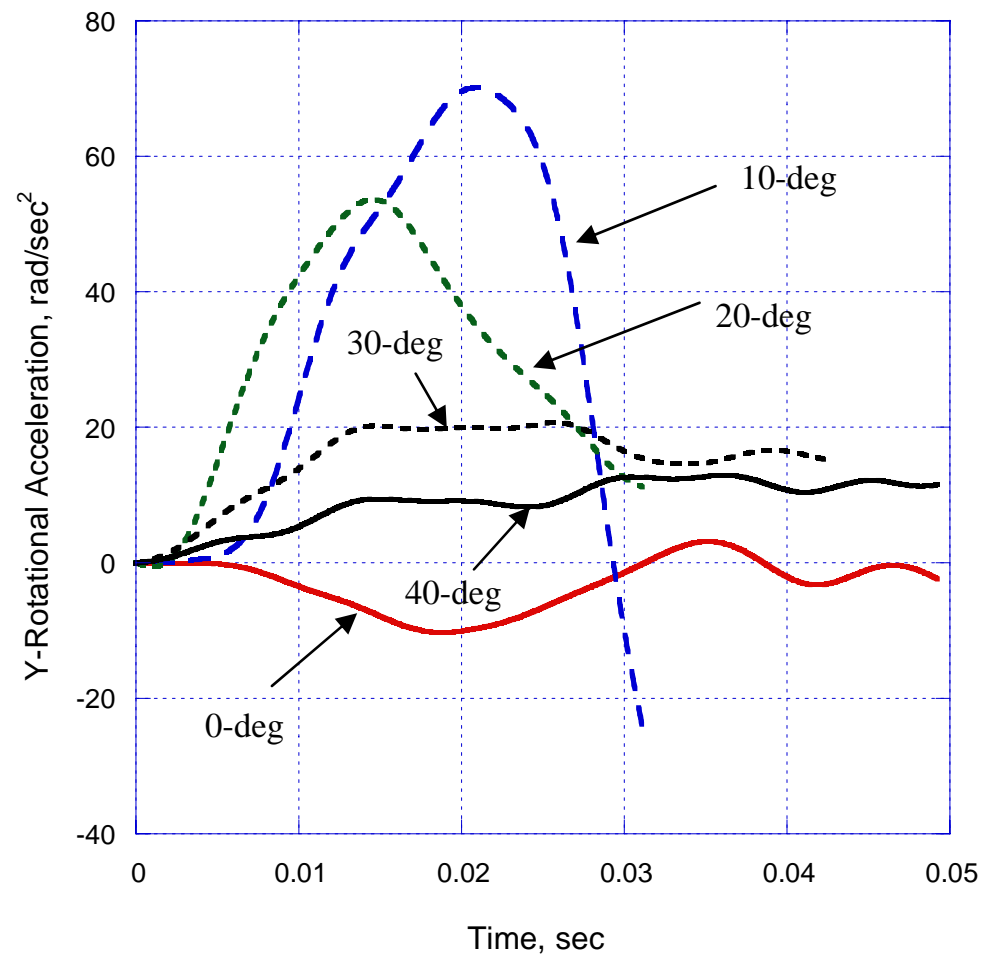

Fig. 21 Filtered Y-rotational acceleration histories for the water landing of the flexible model with various pitch angles. 


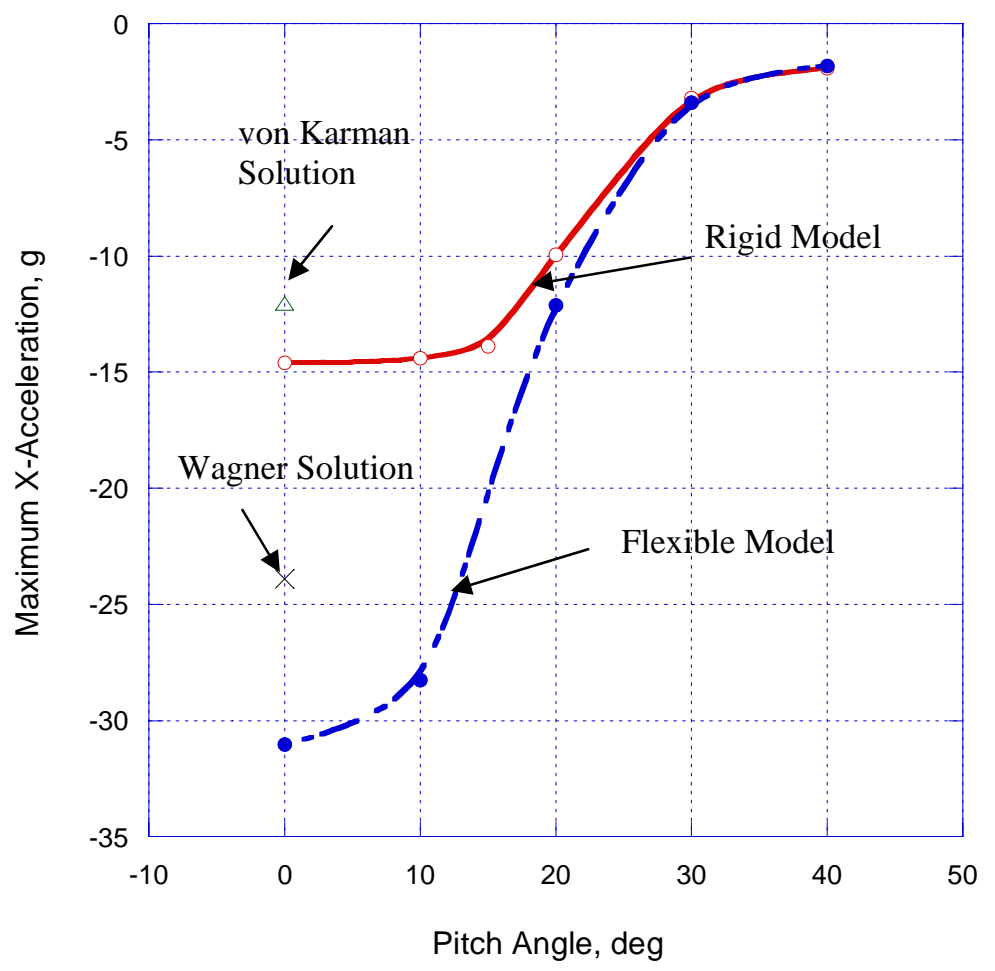

Fig. 22 Maximum $\mathrm{X}$-acceleration as a function of pitch angle for the water landing of the rigid and the flexible models.

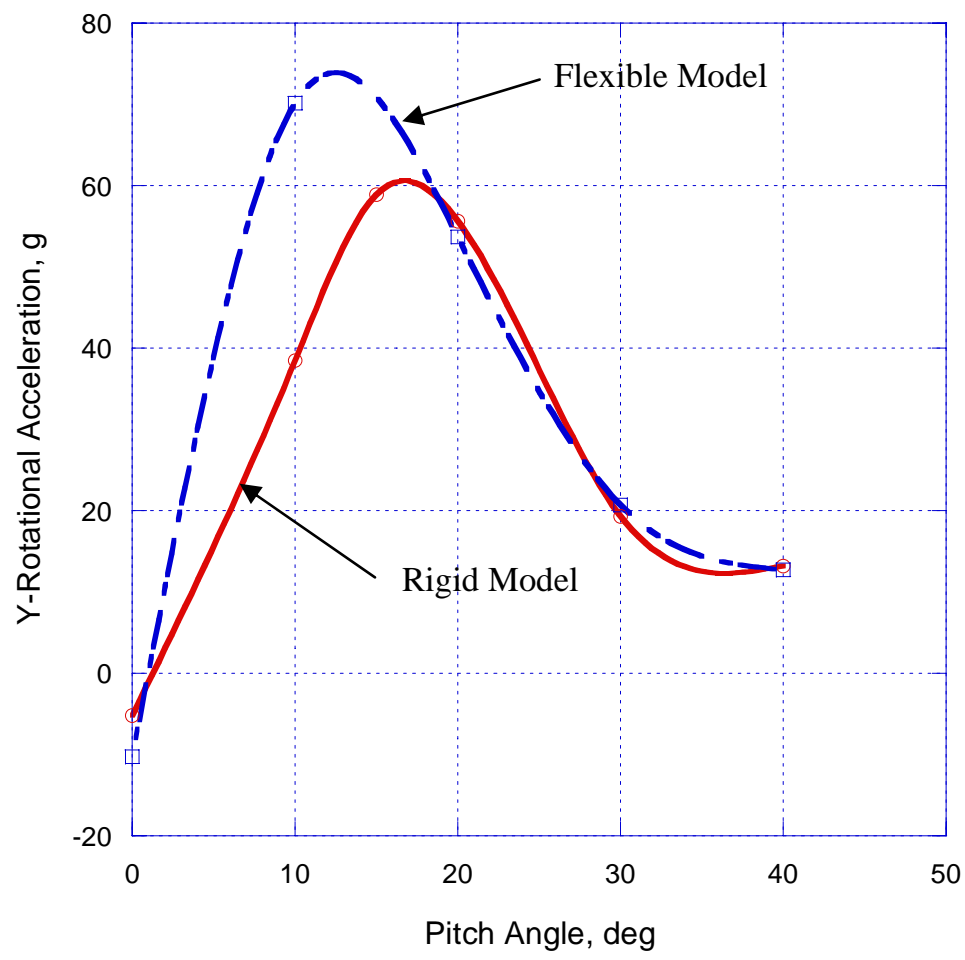

Fig. 23 Maximum Y-rotational acceleration as a function of pitch angle for the water landing of the rigid and the flexible models. 